\title{
SEMIGROUP THEORY APPLIED TO OPTIONS
}

\author{
D. I. CRUZ-BÁEZ AND J. M. GONZÁLEZ-RODRÍGUEZ
}

Received 5 November 2001 and in revised form 5 March 2002

Black and Scholes (1973) proved that under certain assumptions about the market place, the value of a European option, as a function of the current value of the underlying asset and time, verifies a Cauchy problem. We give new conditions for the existence and uniqueness of the value of a European option by using semigroup theory. For this, we choose a suitable space that verifies some conditions, what allows us that the operator that appears in the Cauchy problem is the infinitesimal generator of a $C_{0}$-semigroup $T(t)$. Then we are able to guarantee the existence and uniqueness of the value of a European option and we also achieve an explicit expression of that value.

\section{Introduction}

In this section we recall the basic concepts of options theory [10]. The value of a European call option is a contract verifying that at a prescribed time in the future, known as the expiry date, the owner of the option may purchase a prescribed asset, known as the underlying asset or, briefly, the underlying, for a prescribed amount, known as the exercise price or strike price.

The trading options and their theoretical study have been known for long, but only since the early 1970s they have experimented a spectacular development. The main purpose in option studies is to find a fair arbitrage free price for these instruments. The first solution to the problem was given by Bachelier in 1900 [1, page 17]. However, in the early 1970s a complete option valuation based on equilibrium theoretical hypothesis for speculative prices was finally developed. The works of 
Black and Scholes [3] and Merton [7] were the culmination of this great effort.

In [3], Black and Scholes proved that under certain natural assumptions about the market place, the value of a European option $C$, as a function of the current value of the underlying asset, $x$, and time, $t$ such that $C=C(x, t)$ verifies the following Cauchy problem:

$$
\frac{\partial C}{\partial t}+\frac{1}{2} \sigma^{2} x^{2} \frac{\partial^{2} C}{\partial x^{2}}+r x \frac{\partial C}{\partial x}-r C=0, \quad x \geq 0, t \in[0, T],
$$

with

$$
\begin{gathered}
C(0, t)=0 ; \\
C(x, t) \sim x \text { as } x \longrightarrow \infty ; \\
C(x, T)=C_{T}=\max (x-E, 0), \quad x \in(0, \infty),
\end{gathered}
$$

where the value of the call option also depends on the volatility of the underlying asset $\sigma$, the exercise price $E$, the expiry $T$, and the interest rate $r$, where $r$ and $\sigma$ are constant in this work. Note that the present study is for stocks without dividends.

An explicit solution for (1.1) and (1.2) can be found in [10]. Moreover, in [6] some conditions are established for the existence and uniqueness of the Cauchy problem (1.1) and (1.2), that is,

$$
\begin{gathered}
\frac{\partial C}{\partial t}, \frac{\partial^{k} C}{\partial x^{k}} \in \mathcal{C}((0, \infty) \times(0, T)), \quad k=0,1,2, \\
C(x, t) \in \mathcal{C}((0, \infty) \times[0, T]), \\
|C(x, t)| \leq c_{1} \exp \left(c_{2}(\ln x)^{2}\right),
\end{gathered}
$$

for some $c_{1}, c_{2} \geq 0$, and where $\mathcal{C}$ denotes the continuous functions (see [6, Chapter 2, Theorem 10] for uniqueness and [6, Chapter 9, Theorem 2] for existence).

Our purpose is to obtain new conditions that will guarantee the existence and uniqueness of the Cauchy problem (1.1) and (1.2) using the semigroup theory.

Note that the results that we will obtain are also established for a put option without more than changing the boundary and final conditions of the Cauchy problem, that is,

$$
\begin{gathered}
\frac{\partial P}{\partial t}+\frac{1}{2} \sigma^{2} x^{2} \frac{\partial^{2} P}{\partial x^{2}}+r x \frac{\partial P}{\partial x}-r P=0, \quad x \geq 0, t \in[0, T] \\
P(0, t)=E e^{-r(T-t)}
\end{gathered}
$$




$$
\begin{gathered}
P(x, t) \longrightarrow 0 \quad \text { as } x \longrightarrow \infty ; \\
P(x, T)=\max (E-x, 0), \quad x \in(0, \infty) .
\end{gathered}
$$

We recall that for a non-dividend-paying stock, the value of a European call with a certain exercise price and exercise date can be deduced from the value of a European put with the same exercise price and date, and vice versa (put-call parity).

The paper is organized as follows. In Section 2, we give an introduction to the semigroup theory, and using this theory, in Section 3 we obtain a theorem for existence and uniqueness, besides getting an explicit expression of the European option.

\section{Semigroup theory}

Now, we give an introduction to semigroup theory (see $[2,4,8]$ ). Throughout this section $X$ is a Banach space, with norm $\|\cdot\|$.

Definition 2.1. A one-parameter family $T(t), 0 \leq t<\infty$, of bounded linear operators from $X$ into $X$ is a semigroup of bounded linear operator on $X$ if

(i) $T(0)=I$, (where $I$ is the identity operator on $X$ );

(ii) $T(t+s)=T(t) T(s)$ for every $t, s \geq 0$ (the semigroup property).

A semigroup of bounded linear operators, $T(t)$, is uniformly continuous if

$$
\lim _{t \rightarrow 0}\|T(t)-I\|=0 .
$$

From the definition, it is clear that if $T(t)$ is a uniformly continuous semigroup of bounded linear operators, then

$$
\lim _{x \rightarrow t}\|T(x)-T(t)\|=0 .
$$

The linear operator $A$, defined by

$$
\begin{gathered}
D(A)=\left\{x \in X: \lim _{t \rightarrow 0} \frac{T(t) x-x}{t} \text { exists }\right\}, \\
A x=\lim _{t \rightarrow 0} \frac{T(t) x-x}{t}=\left.\frac{d^{+} T(t) x}{d t}\right|_{t=0},
\end{gathered}
$$

is the infinitesimal generator of the semigroup $T(t), D(A)$ is the domain of $A$. 
134 Semigroup theory applied to options

THEOREM 2.2. A linear operator $A$ is the infinitesimal generator of a uniformly continuous semigroup if and only if $A$ is a bounded linear operator.

From Definition 2.1, it is clear that a semigroup $T(t)$ has a unique infinitesimal generator. When $T(t)$ is uniformly continuous, its infinitesimal generator is a bounded linear operator.

Definition 2.3. A semigroup $T(t), 0 \leq t<\infty$, of bounded linear operators on $X$ is a strongly continuous semigroup of bounded linear operators if

$$
\lim _{t \rightarrow 0} T(t) x=x,
$$

for every $x \in X$.

A strongly continuous semigroup of bounded linear operators on $X$ will be called a semigroup of class $C_{0}$ or simply a $C_{0}$-semigroup.

Now, we recall that if $A$ is a linear, not necessarily bounded, operator in $X$, the resolvent set $\rho(A)$ of $A$ is the set of all complex numbers $\lambda$ for which $\lambda I-A$ is invertible, that is, $(\lambda I-A)^{-1}$ is a bounded operator in $X$. The family $R(\lambda: A)=(\lambda I-A)^{-1}, \lambda \in \rho(A)$, of bounded linear operators is called the resolvent of $A$.

The following theorem is very important in what follows and it is known as Hille-Yosida's theorem.

Theorem 2.4. A linear (unbounded) operator $A$ is the infinitesimal generator of a $C_{0}$-semigroup of contractions $T(t), t \geq 0$, if and only if

(i) $A$ is closed and $\overline{D(A)}=X$;

(ii) the resolvent set $\rho(A)$ of $A$ contains $\mathbb{R}^{+}$and for every $\lambda>0$,

$$
\|R(\lambda: A)\| \leq \frac{1}{\lambda} .
$$

As a consequence of this theorem we obtain the following corollary.

Corollary 2.5. A linear operator $A$ is the infinitesimal generator of a $\mathrm{C}_{0^{-}}$ semigroup satisfying $\|T(t)\| \leq e^{w t}$ if and only if

(i) $A$ is closed and $\overline{D(A)}=X$;

(ii) the resolvent set $\rho(A)$ of $A$ contains the ray $\{\lambda: \operatorname{Im} \lambda=0, \lambda>w\}$ and for every $\lambda>0$,

$$
\|R(\lambda: A)\| \leq \frac{1}{\lambda-w}
$$


The previous results allow us to prove the following theorem especially useful in what follows.

THEOREM 2.6. Let $A$ be a densely defined linear operator with a nonempty resolvent set $\rho(A)$. The initial value

$$
\frac{d u(t)}{d t}=A u(t), \quad t>0, \quad u\left(t_{0}\right)=x \quad(x \in X)
$$

has a unique solution $u(t)$, which is continuously differentiable on $[0, \infty)$, for every initial value $x \in D(A)$ if and only if $A$ is the infinitesimal generator of a $C_{0}$-semigroup $T(t)$.

\section{A European call option}

As we have seen in the introduction, the Black-Scholes equation and boundary conditions for a European call option with value $C(x, t)$ are

$$
\frac{\partial C}{\partial t}=-\frac{1}{2} \sigma^{2} x^{2} \frac{\partial^{2} C}{\partial x^{2}}-r x \frac{\partial C}{\partial x}+r C, \quad(x, t) \in[0, \infty) \times(0, T),
$$

with

$$
\begin{gathered}
C(0, t)=0 ; \\
C(x, t) \sim x \text { as } x \longrightarrow \infty \\
C(x, T)=C_{T}=\max (x-E, 0), \quad x \in(0, \infty) .
\end{gathered}
$$

Note that the linear differential operator

$$
\frac{\partial}{\partial t}+\frac{1}{2} \sigma^{2} x^{2} \frac{\partial^{2}}{\partial x^{2}}-r x \frac{\partial}{\partial x}-r
$$

has a financial interpretation as a measure of the difference between the return hedged option portfolio (the first two terms) and the return on a bank deposit (the last two terms).

The main objective, in this section, includes guaranteeing the existence and uniqueness of the solution of (3.1) and (3.2), and, furthermore, obtaining an exact expression of the solution.

Consider the space $X^{\alpha}$ defined by

$$
X^{\alpha}=\left\{f: x^{\alpha+1} f(x) \in \mathcal{C}(0, \infty),\|f\|_{\infty}^{\alpha}<\infty\right\},
$$

where $\|\cdot\|_{\infty}^{\alpha}$ is the norm

$$
\|f\|_{\infty}^{\alpha}=\sup _{0 \leq x<\infty}\left|x^{\alpha+1} f(x)\right| .
$$


The space $\left(X^{\alpha},\|\cdot\|_{\infty}^{\alpha}\right)$ is a Banach space.

We define the operator $A: D(A) \subset X^{\alpha} \rightarrow X^{\alpha}$ such that

$$
f(x) \longrightarrow(A f)(x)=-\frac{1}{2} \sigma^{2} x^{2} \frac{d^{2} f}{d x^{2}}-r x \frac{d f}{d x}+r f(x)
$$

being $D(A)=\left\{f \in X^{\alpha}: A f \in X^{\alpha}\right\}$.

Then, we can establish the following result.

THEOREM 3.1. The differential operator $A$ is the infinitesimal generator of a $C_{0}$-semigroup $T(t)$.

Proof. We denote by $\mathcal{C}_{0, \alpha}^{\infty}(\alpha \in \mathbb{R})$, the space $\mathcal{C}_{0, \alpha}^{\infty}=\left\{f: x^{\alpha+1} f(x) \in \mathcal{C}_{0}^{\infty}\right\}$. Then we can see that $A$ is a closed operator and $D(A)$ is dense in $X^{\alpha}$ because $D(A)$ contains $\mathcal{C}_{0, \alpha}^{\infty}$ which is dense in $X^{\alpha}$.

Therefore we should study the resolvent set $\rho(A)$. We must find a function $g$ in $D(A)$ such that

$$
(\lambda I-A) g(x)=f(x),
$$

that is,

$$
(\lambda-r) g(x)+r x \frac{d g}{d x}+\frac{1}{2} \sigma^{2} x^{2} \frac{d^{2} g}{d x^{2}}=f(x)
$$

that we write in the following way

$$
(\lambda-r) g(x)+\left(r-\frac{1}{2} \sigma^{2}\right) x \frac{d g}{d x}+\frac{1}{2} \sigma^{2}\left(x \frac{d}{d x}\right)^{2} g(x)=f(x)
$$

To solve (3.9) we use the Mellin transform, that is,

$$
\mathcal{M}(g(x))(s)=\int_{0}^{\infty} x^{s-1} g(x) d x \quad(\operatorname{Re} s>0)
$$

and we take into account (see [5, (11), page 307]) that

$$
\begin{gathered}
\mathcal{M}\left(\left(x \frac{d}{d x}\right)^{2} g(x)\right)(s)=s^{2} G(s), \\
M\left(x \frac{d}{d x} g(x)\right)(s)=-s G(s),
\end{gathered}
$$

where $G(s)=\operatorname{Mg} g(s)$. 
By (3.11), (3.9) transforms to

$$
(\lambda-r) G(s)-\left(r-\frac{1}{2} \sigma^{2}\right) s G(s)+\frac{1}{2} \sigma^{2} s^{2} G(s)=F(s)
$$

obtaining $G(s)$

$$
\begin{aligned}
G(s) & =\frac{F(s)}{(1 / 2) \sigma^{2} s^{2}-\left(r-(1 / 2) \sigma^{2}\right) s-r+\lambda} \\
& =\frac{2}{\sigma^{2}} F(s) \cdot \frac{1}{s^{2}-\left(2 r / \sigma^{2}-1\right) s-\left(2(r-\lambda) / \sigma^{2}\right)} \\
& =\frac{2}{\sigma^{2}} F(s) \cdot \frac{1}{\left(s-s_{1}\right)\left(s-s_{2}\right)}
\end{aligned}
$$

where $s_{1,2}=r / \sigma^{2}-1 / 2 \pm \sqrt{\left(r / \sigma^{2}+1 / 2\right)^{2}-2 \lambda}=\alpha \pm \sqrt{\beta^{2}-2 \lambda}$, being $\alpha=$ $r / \sigma^{2}-1 / 2 ; \beta=\left(r / \sigma^{2}+1 / 2\right)^{2}$.

We consider the case $r>(1 / 2) \sigma^{2}$, the other case is proved in a similar way using [5, (9), page 342].

If we apply in (3.13) the inverse Mellin transform, we have by virtue of $[5,(7)$, page 341]

$$
g(x)= \begin{cases}f(x) *\left\{\left(s_{1}-s_{2}\right)^{-1} \cdot\left(x^{s_{1}}-x^{s_{2}}\right)\right\}, & 0<x<1 \\ 0, & 1<x<\infty\end{cases}
$$

where $*$ represents the Mellin convolution [9], that is,

$$
f * g(x)=\int_{0}^{\infty} f\left(\frac{x}{y}\right) g(y) \frac{1}{y} d y .
$$

Then, for $0<y<1$,

$$
\begin{aligned}
g(x) & =R(z: A) f \\
& =\frac{\sqrt{\beta^{2}-2 \lambda}}{\sigma^{2}\left(2 \lambda-\beta^{2}\right)} \int_{0}^{\infty} f\left(\frac{x}{y}\right) \cdot \frac{1}{y}\left[y^{-\alpha+\sqrt{\beta^{2}-2 \lambda}}-y^{-\alpha-\sqrt{\beta^{2}-2 \lambda}}\right] d y .
\end{aligned}
$$

On the other hand, using (3.16) and making the change of variables $x / y=u$ gives 
138 Semigroup theory applied to options

$$
\begin{aligned}
\left|x^{\alpha+1} g(x)\right| \leq c_{1}\left|\frac{\sqrt{\beta^{2}-2 \lambda}}{\sigma^{2}}\right| \frac{1}{2 \lambda-\beta^{2}}\|f\|_{\infty}^{\alpha} \\
\cdot\left|x\left(\int_{x}^{\infty}\left(\frac{x}{u}\right)^{\sqrt{\beta^{2}-2 \lambda}} u^{-2} d u-\int_{x}^{\infty}\left(\frac{x}{u}\right)^{-\sqrt{\beta^{2}-2 \lambda}} u^{-2} d u\right)\right|,
\end{aligned}
$$

where $c_{1}$ is a positive constant.

Thus, evaluating the two integrals we obtain

$$
\left|x^{\alpha+1} g(x)\right| \leq c_{2} \frac{1}{\lambda-\beta^{2} / 2}\|f\|_{\infty}^{\alpha}
$$

and therefore,

$$
\|g\|_{\infty}^{\alpha} \leq c_{2} \frac{1}{\lambda-\beta^{2} / 2}\|f\|_{\infty}^{\alpha}
$$

where $c_{2}$ is a positive constant and $\operatorname{Re} \lambda>\beta^{2} / 2=(1 / 2)\left(r / \sigma^{2}+1 / 2\right)^{4}$.

Then by Corollary $2.5, A$ generates a $C_{0}$-semigroup $T(t)$, where

$$
\begin{aligned}
& T(t) f(x) \\
& =\frac{1}{2 \pi i} \int_{c-i \infty}^{c+i \infty} e^{\lambda x}[R(\lambda: A) f](x) d \lambda \\
& \left.=\frac{1}{2 \pi i \sigma^{2}} \int_{c-i \infty}^{c+i \infty} e^{\lambda x} \cdot\left(\int_{x}^{\infty} f(u) x^{-\alpha-1} u^{\alpha+1}\right) \frac{x}{u^{2}} d u\right) \frac{\sqrt{\beta^{2}-2 \lambda}}{\left(2 \lambda-\beta^{2}\right)} d \lambda, \\
& \cdot\left(\left(\frac{x}{u}\right)^{\sqrt{\beta^{2}-2 \lambda}}-\left(\frac{x}{u}\right)^{-\sqrt{\beta^{2}-2 \lambda}}\right.
\end{aligned}
$$

for $\operatorname{Re} \lambda>\beta^{2} / 2$.

By Theorems 2.6 and 3.1, we can guarantee the existence and uniqueness of problem (3.1) with conditions (in this case, $C \in X^{\alpha}$ ) different from those given in [6] and obtain the explicit expression in a way different from [10, Chapter 5, pages 97-100], that is, we have the following theorem. 
THEOREM 3.2. There is a unique solution $C(x, t)$ of (3.1) and (3.2) in the space $X^{\alpha}$, and

$$
C(x, t)=T(t) C_{T}
$$

\section{Acknowledgment}

The authors are thankful to the referee for the valuable comments that led to the improvement of this paper.

\section{References}

[1] L. Bachelier, Theory of speculation, The Random Character of Stock Market Prices (P. Cootner, ed.), MIT Press, Massachusetts, 1964, pp. 17-78.

[2] A. Belleni-Morante, Applied Semigroups and Evolution Equations, Oxford Mathematical Monographs, Oxford University Press, New York, 1979.

[3] F. Black and M. Scholes, The pricing of options and corporate liabilities, J. Polit. Econ. 81 (1973), 637-654.

[4] H. Brézis, M. G. Crandall, and F. Kappel (eds.), Semigroups, Theory and Applications. Vol. II, Pitman Research Notes in Mathematics, vol. 152, Longman Scientific \& Technical, Harlow, 1986.

[5] A. Erdélyi, W. Magnus, F. Oberhettinger, and F. G. Tricomi, Tables of Integral Transforms. Vol. I, McGraw-Hill, New York, 1954.

[6] A. Friedman, Partial Differential Equations of Parabolic Type, Robert E. Krieger Publishing, New York, 1983.

[7] R. C. Merton, Theory of rational option pricing, Bell J. Econom. Management Sci. 4 (1973), 141-183.

[8] A. Pazy, Semigroups of Linear Operators and Applications to Partial Differential Equations, Applied Mathematical Sciences, vol. 44, Springer-Verlag, New York, 1983.

[9] I. N. Sneddon, Fourier Transforms, McGraw-Hill, New York, 1951.

[10] P. Wilmott, Paul Wilmott introduces Quantitative Finance, John Wiley \& Sons, New York, 2001.

D. I. Cruz-Báez: Department of Applied Economics, University of La Laguna, Campus de Guajara, s/n, Edificio de Económicas - Empresariales, 38071 La Laguna (Tenerife), Spain

E-mail address: dicruz@ull.es

J. M. González-Rodríguez: Department of Applied Economics, University of La Laguna, Campus de Guajara, s/n, Edificio de Económicas-Empresariales, $38071 \mathrm{La}$ Laguna (Tenerife), Spain

E-mail address: jomagon@ull.es 


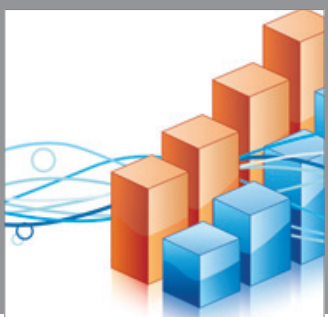

Advances in

Operations Research

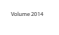

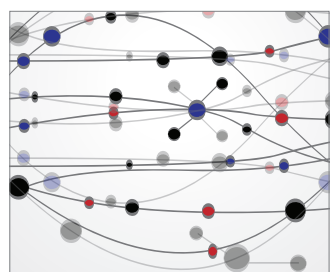

\section{The Scientific} World Journal
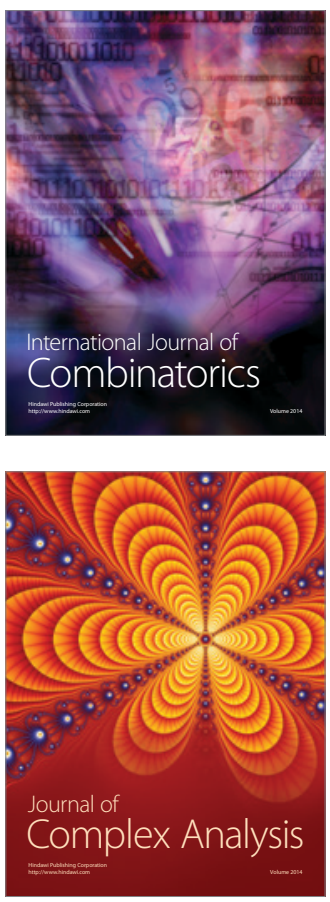

International Journal of

Mathematics and

Mathematical

Sciences
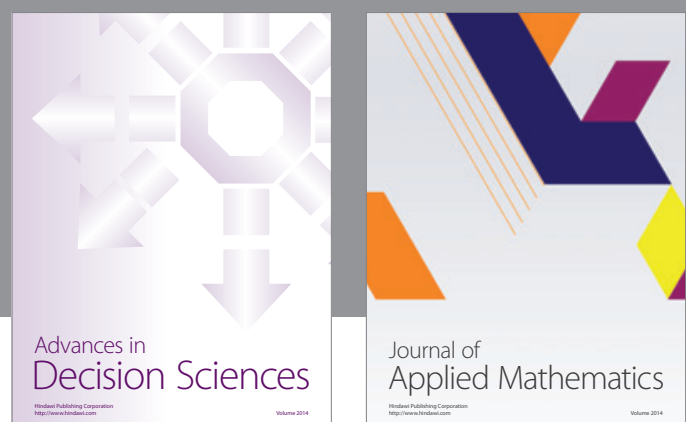

Journal of

Applied Mathematics
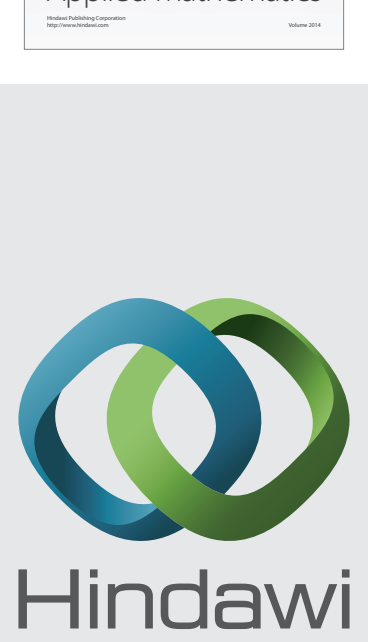

Submit your manuscripts at http://www.hindawi.com
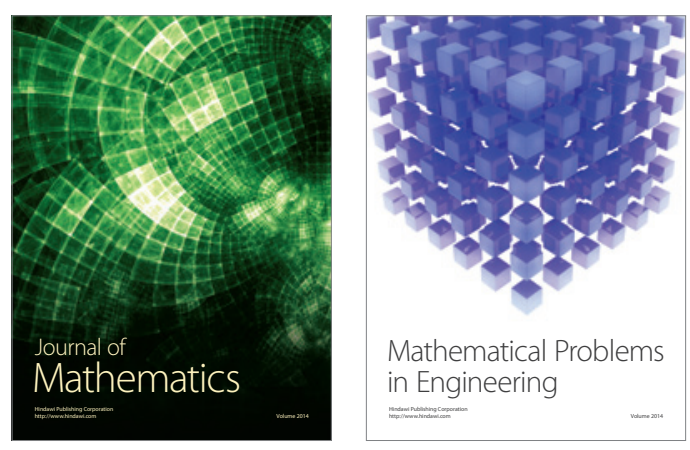

Mathematical Problems in Engineering
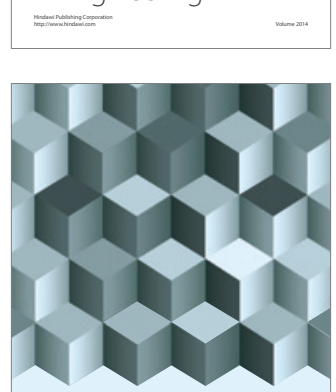

Journal of

Function Spaces
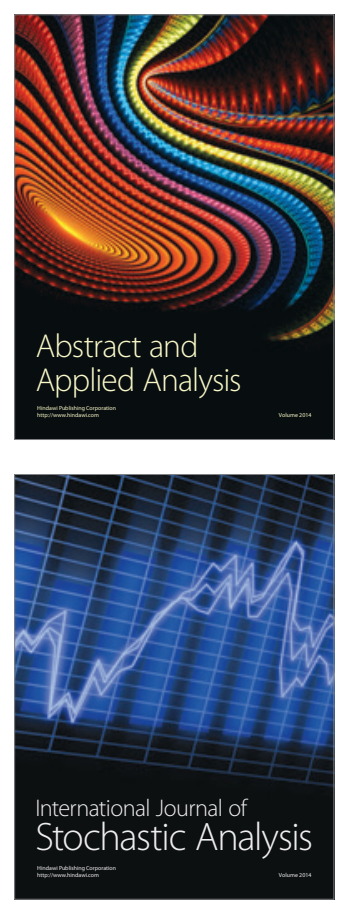

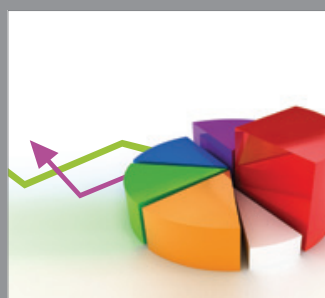

ournal of

Probability and Statistics

Promensencen
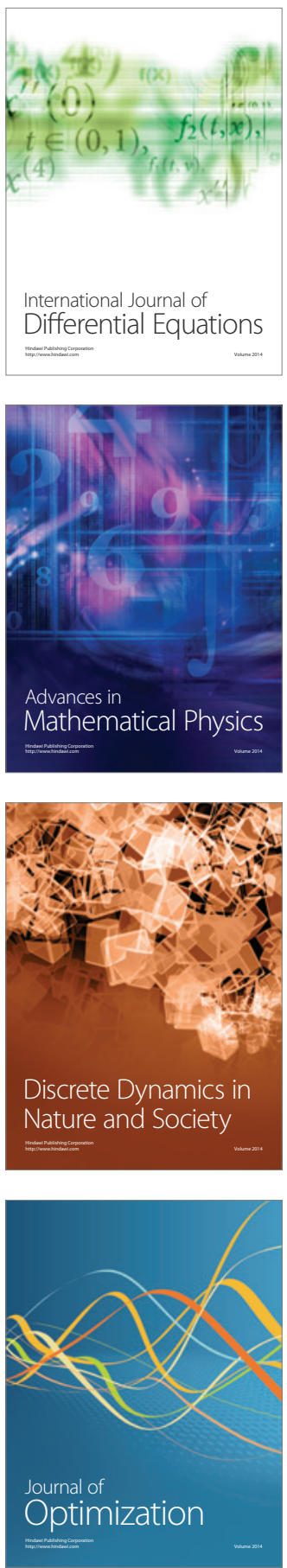